Iranian Journal of Pathology | ISSN: 2345-3656

\title{
Hepatoid Variant of Yolk Sac Tumor of Both Ovaries With Widespread Intra- abdominal and Lung Metastasis: A Case Report
}

\author{
Srilatha Parampalli Srinivas ${ }^{1 *}$, Archana Shivamurthy ${ }^{2}$, Lakshmi Rao ${ }^{3}$, Rajeshwari Gurumoorthy Bhat ${ }^{4}$ \\ 1. Dept . of Pathology, Kasturba Medical College, Manipal Academy of Higher Education, Manipal, Karnataka, \\ India \\ 2. Dept. of Pathology, Melaka Manipal Medical College, Manipal Academy of Higher Education, Manipal, \\ Karnataka, India \\ 3. Dept. of Pathology, Armed Forces Hospital, Muscat, Oman \\ 4. Dept. of Obstetrics and Gynaecology, Melaka Manipal Medical College, Manipal Academy of Higher Educa- \\ tion, Manipal, Karnataka, India
}

\begin{tabular}{c} 
KEYWORDS \\
\hline Neoplasm; \\
Endodermal Sinus Tumour; \\
Female; \\
Ovary \\
\hline
\end{tabular}

\section{Article Info}

Received 30 Dec 2016; Accepted 05 April 2018; Published Online 17 July 2018;

\section{ABSTRACT}

Hepatoid variant of yolk sac tumor of ovary is an unusual tumor with an aggressive behavior. It is usually observed in young females, presents with abdominal complaints and is associated with raised $\alpha$-fetoprotein (AFP) levels. It should be differentiated from other hepatoid tumors involving the ovary. A complete patient evaluation with gross, microscopy, and immunohistochemistry can identify the site of origin to administer appropriate treatment.

The current study reported the case of a 30-year-old married parous female presenting with abdominal distention and pain of two months duration. She had regular menstrual cycles. Based on lab investigations her serum AFP level was markedly raised to $34,244 \mathrm{ng} / \mathrm{mL}$ (normal range: $0-9 \mathrm{ng} / \mathrm{mL}$ ). Computerized tomography (CT) scan showed large lobulated heterogeneous mass in both ovaries and omental, gall bladder, and lung metastasis. A CT guided biopsy of the ovarian mass was done. On histopathology, a differential diagnosis of hepatoid variant of yolk sac tumor, hepatoid carcinoma of ovary and hepatoid tumor arising from gall bladder metastasizing to the ovary were observed. Patient underwent surgery. Per operatively gross ascites with bilateral ovarian mass, extensive omental, pelvic, and gall bladder deposits were observed. Bilateral salpingo-oophorectomy with omental deposit biopsy was conducted. Histopathology along with immunohistochemistry confirmed a diagnosis of hepatoid variant of yolk sac tumor in both ovaries with widespread intra-abdominal metastasis.

Dr. Srilatha Parampalli Srinivas, MD (Pathology), Associate Professor, Dept . of Pathology, Kasturba Medical College, Manipal Academy of Higher Education, Manipal, Karnataka, India E-mail- drsrilatha2003@ hotmail.com

Corresponding information:

Copyright (C) 2018, IRANIAN JOURNAL OF PATHOLOGY. This is an open-access article distributed under the terms of the Creative Commons Attribution-noncommercial 4.0 International License which permits copy and redistribute the material just in noncommercial usages, provided the original work is properly cited.

\section{Introduction}

Hepatoid variant of yolk sac tumor of ovary is a rare neoplasm of germ cell origin. It is usually observed in young females, presents with abdominal complaints and is associated with raised serum $\alpha$-fetoprotein (AFP) levels (1). It may be associated with amenorrhea, hirsutism, and virilism (2) or gonadal dysgenesis $(2,3)$. It should be differentiated from primary hepatoid carcinoma of ovary, metastatic deposits of hepatocellular carcinoma, and metastatic deposits of hepatoid carcinoma occurring in other organs such as pancreas, mediastinum, stomach, urinary bladder, lung, and kidney $(1,4)$. The current study reported a case of hepatoid yolk sac tumor of both ovaries with widespread intra-abdominal and lung metastasis in a 30-year-old parous female.

\section{Case Report}

A 30-year-old married parous female presented with abdominal distention and pain of two months duration. She had history of early satiety, loss of appetite, and loose stools after food intake. She had regular menstrual cycles. Her abdomen was distended with ascites. The vaginal examination revealed a solid 
mass in the posterior fornix. Computerized tomography (CT) scan showed multiple heterogeneously enhancing lesions in the bilateral adnexal regions not observed separately from bilateral ovaries with multiple omental, peritoneal, sub-diaphragmatic, subhepatic, gall bladder, pouch of Douglas, and lung metastasis. No liver mass was noted. A CT guided biopsy of the ovarian mass was done. Based on lab investigations her serum AFP level was 34,244 ng/mL (normal range: $0-9 \mathrm{ng} / \mathrm{mL}$ ) and CA125 level was $77 \mathrm{ng} /$ $\mathrm{mL}$ (normal range: 0-35 ng/mL). In histopathology, a differential diagnosis of hepatoid variant of yolk sac tumor, hepatoid carcinoma of ovary, and hepatoid tumor arising from gall bladder metastasizing to the ovary were observed.

Patient underwent a staging laparotomy. In the operation seven liters of ascitic fluid was drained. Bilateral ovarian mass, extensive omental caking, pelvis, pouch of Douglas, and gall bladder deposits were observed. Bilateral salpingo-oophorectomy with omental deposit biopsy was conducted. Since gall bladder with deposits was friable, it was not touched to avoid injury. Grossly, both ovaries were enlarged with grey white solid, cystic, and hemorrhagic areas (Figure 1).

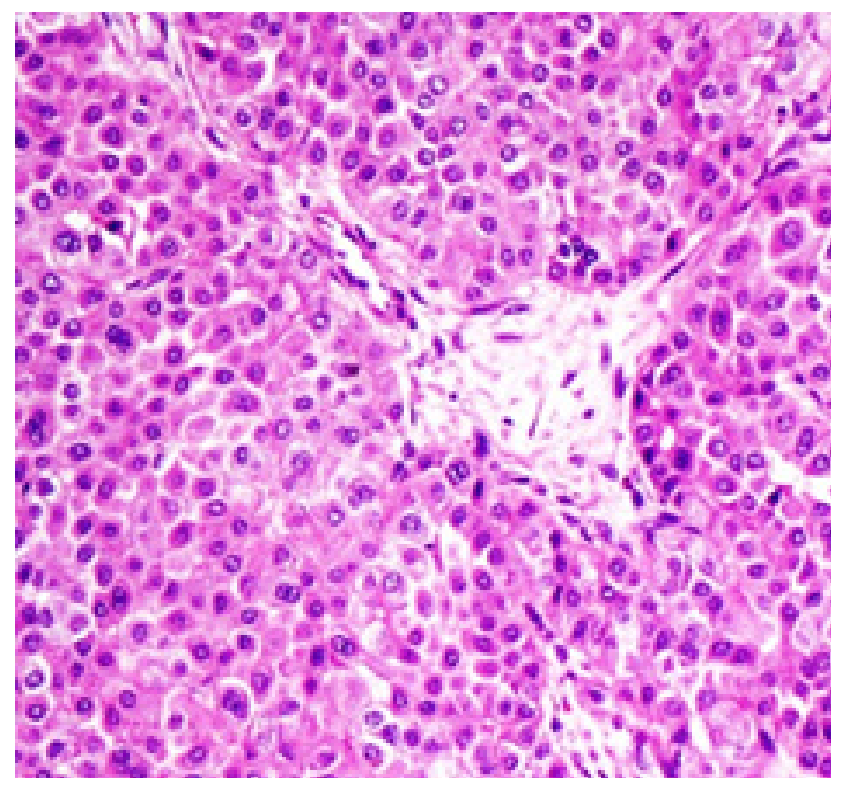

Figure 2. Histological section showing tumor arranged in discrete masses and broad bands of large eosinophilic polygonal cells separated by fibrous stroma (H\&E; 200X)
Histopathology of both ovaries and omental deposit showed sheets of large polygonal cells interspersed by fibrous bands (Figure 2).

Tumor cells had dense eosinophilic to focally vacuolated cytoplasm, vesicular nucleus with prominent nucleoli, and few mitotic figures. Many intra- and extra- cellular hyaline globules, scattered syncytiotrophoblastic giant cells (Figure 3), microcytic spaces, and focal glandular elements (Figure 4) were observed.

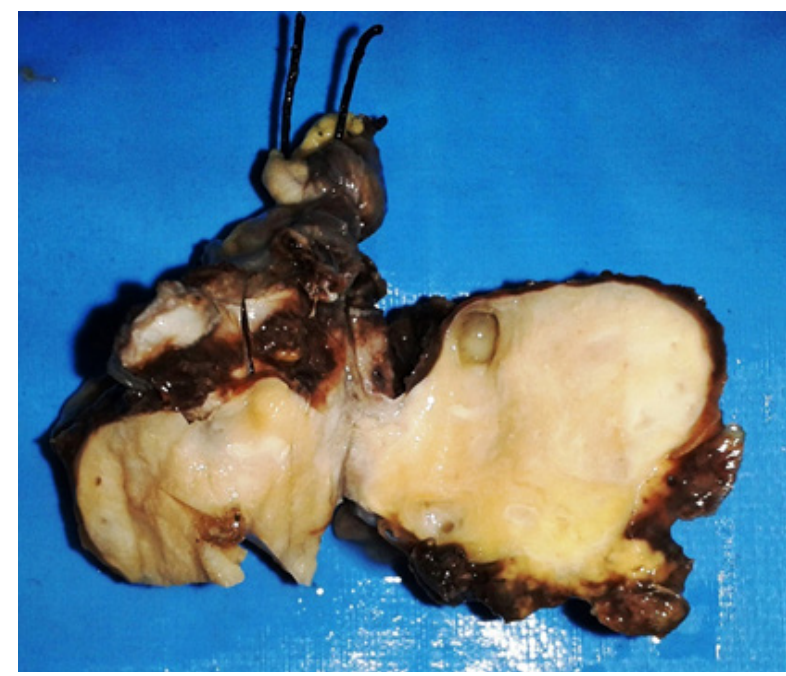

Figure 1. Gross picture of one of the ovaries showing solid and cystic areas

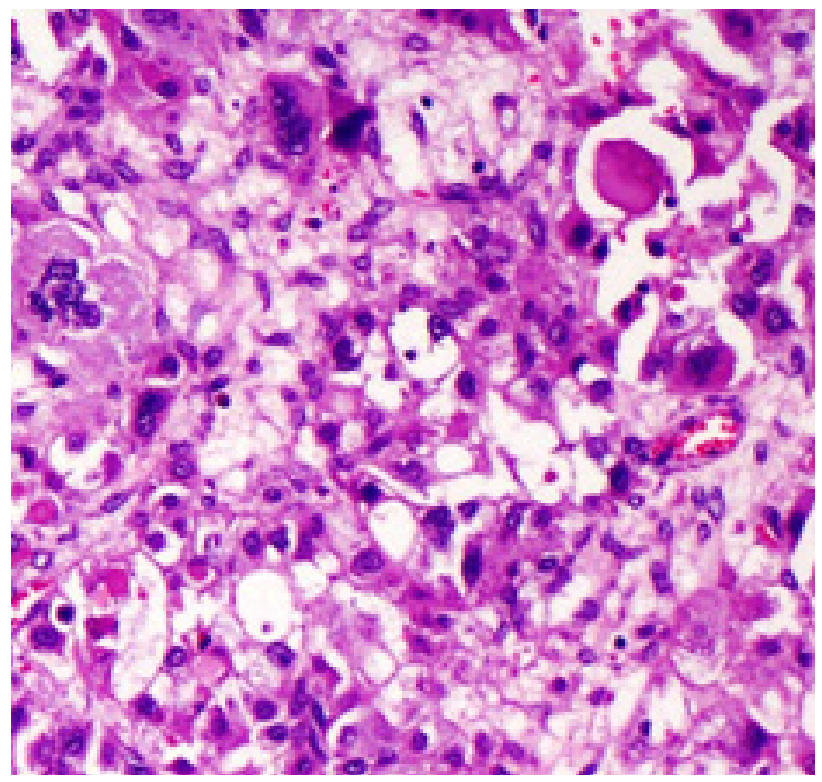

Figure3. Histological section showing tumor with intraand extra-cellular hyaline globules and scattered syncytiotrophoblastic (H\&E; 200X) 


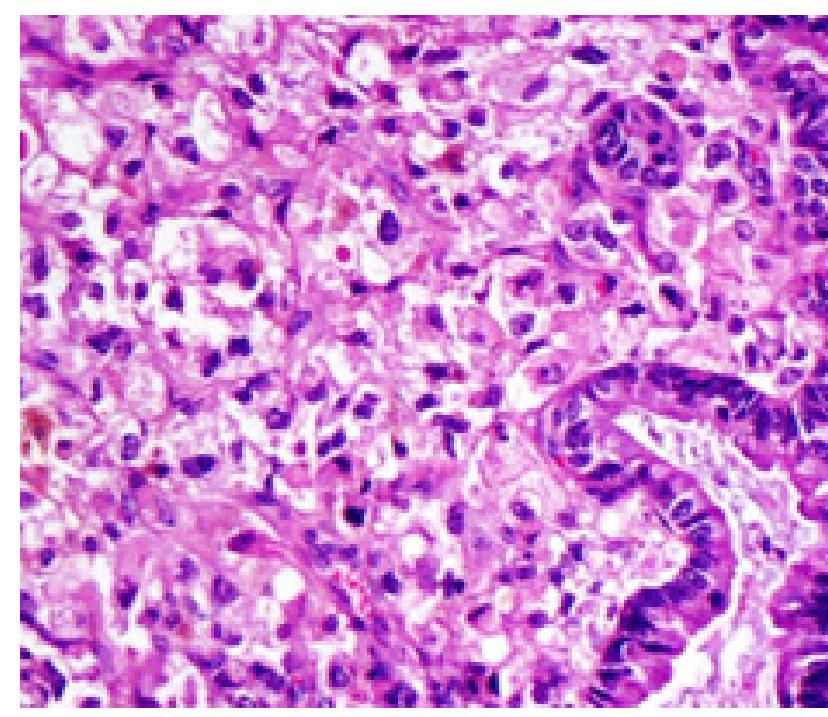

Figure 4. Histological section showing tumor with glandular elements (H\&E; 200X)

Based on immunohistochemistry, pancytokeratin (AE1/AE3) was focally positive in the tumor cells. CK7 was focally faintly positive in the tumor cells. AFP was positive in the intra- and extra- cellular hyaline globules. Hep Par1 was diffusely positive in the tumor cells (Figure 5).

A final diagnosis of hepatoid variant of yolk sac tumor of both ovaries with widespread intra- abdominal and lung metastasis was rendered. Since she was in stage IV, palliative cisplatin-based chemotherapy regimen comprising three courses of bleomycin, etoposide and cisplatinum and one course of cisplatinum and etoposide at three-week interval was planned. During the course of chemotherapy, she developed liver metastasis and pleural effusion. After the fourth course of chemotherapy, since the AFP levels did not reach the reference range, she was given three more course of chemotherapy. After the seventh course of chemotherapy, presently her AFP level was $53 \mathrm{ng} /$ $\mathrm{mL}$. She tolerated the courses of chemotherapy well.

\section{Discusstion}

In 1982, Prat et al., first described seven cases of unusual types of yolk sac tumors with predominant pattern resembling hepatocellular carcinoma and termed them as hepatoid yolk sac tumor of the ovary (endodermal sinus tumor with hepatoid differentiation). He
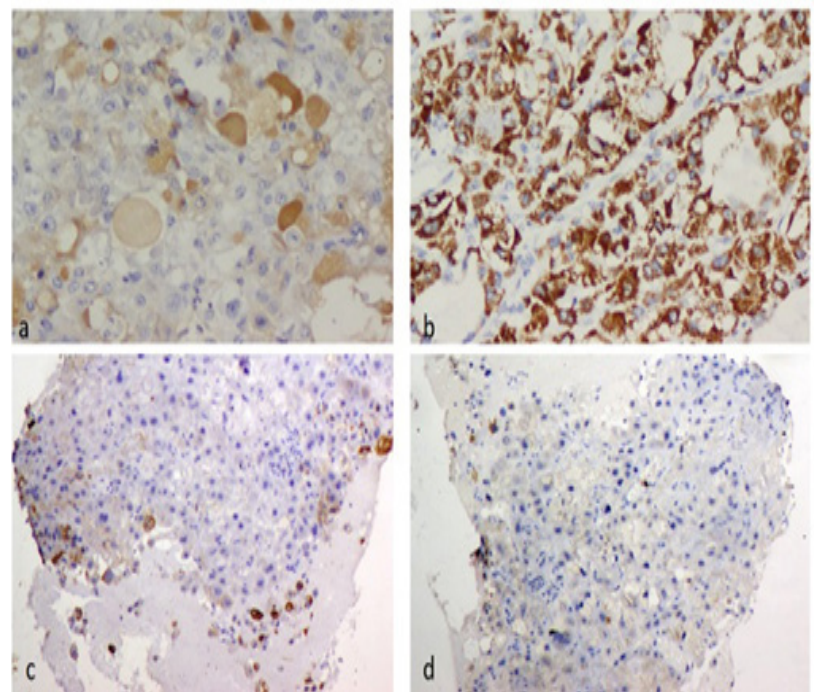

Figure 5. Immunohistochemistry showing intra- and extra- cellular hyaline globules positive for AFP (a), diffuse positivity for Hep Par1 (b), focal positivity for pancytokeratin (c), and focal faint positivity for CK7 (d) (200X)

also proposed the embryologic basis behind this differentiation. In early embryonic life, the yolk sac is in direct continuity with the primitive gut, which is also referred to as secondary yolk sac vesicle. From this vesicle, the hepatobiliary analage originates (5). Histologically, this tumor is characterized by discrete masses, nests and broad bands of large polygonal cells separated by fibrous stroma. The tumor cells have abundant eosinophilic, granular cytoplasm, and central round nuclei with prominent nucleoli. Numerous intra- and extra-cellular periodic acid- Schiff (PAS) positive, diastase resistant hyaline globules are observed. Occasional glandular elements can be observed $(2,5)$

Hepatoid ovarian yolk sac tumor should be differentiated from primary hepatoid carcinoma of ovary, metastatic deposits of hepatocellular carcinoma to the ovary, and metastatic deposits of hepatoid carcinoma occurring in other organs such as pancreas, mediastinum, stomach, gall bladder, urinary bladder, lung, and kidney to the ovary.

Primary hepatoid carcinoma of ovary has supposedly a surface epithelial origin. It is usually observed in the post-menopausal women aged 42 to 78 years (mean 63), with normal gonadal development, increase in serum AFP levels and CA 125 and presence of intracellular hyaline globules. This tumor is posi- 
tive for pancytokeratin, Hep Par1, and diffusely positive for polyclonal CEA, AFP, and CK7.

Hepatoid ovarian yolk sac tumors is a germ cell tumor usually observed in younger population aged 7 to 54 years (mean 22), can be associated with gonadal dysgenesis and virilising symptoms, increase in serum AFP levels and CA125, presence of intra- and extra-cellular hyaline globules, occasional presence of glandular structures, and has an aggressive course. This tumor is positive for pancytokeratin, AFP, focally positive for polyclonal CEA and negative for CK7 $(2,6-8)$. Some of them can be positive for Hep Par1 (9).

Tumors metastatic to the ovary constitute about $5 \%$ $10 \%$ of all ovarian tumors (10). Primary hepatocellular carcinoma, and hepatoid carcinomas arising from organs such as pancreas (11), mediastinum, stomach, urinary bladder, gall bladder (12), lung, and kidney $(3,6)$ can rarely metastasis to the ovary.

In the current case, the patient was young and presented with widespread metastasis, very high serum AFP levels, elevated serum CA 125 levels, with presence of both intra- and extra-cellular hyaline globules, focal glandular elements, and few scattered syncytiotrophoblasts. In the current case, the tumor showed focal faint positivity for CK7, focal positivity for pancytokeratin (AE1/AE3), AFP positive hyaline globules, and diffusion positivity for Hep Par 1. Absence of bile in the tumor cells and no liver lesions at the time of diagnosis ruled out metastatic deposits of hepatocellular carcinoma to the ovary.

In patients with advanced disease, such as the current case, a surgical debulking of the tumor followed by palliative cisplatin-based chemotherapy improves the prognosis. The current study patient completed seven courses of cisplatin-based chemotherapy and her serum $\alpha$-fetoprotein levels gradually reduced.

\section{Conclusion}

Hepatoid variant of yolk sac tumor is a rare and aggressive tumor. It should be differentiated from other hepatoid tumors involving the ovary. A complete patient evaluation with radiology, gross, microscopy, and immunohistochemistry can identify the site of origin to administer appropriate treatment.

\section{Acknowledgement}

There was no financial or material support for the study.

\section{Conflict of interest}

The authors declared no conflict of interest.

\section{References}

1. Devouassoux-Shisheboran M, Schammel DP, Tavassoli FA. Ovarian hepatoid yolk sac tumors : morphological, immunohistochemical and ultrastructural features . Histopathology. 1999;34(5):462-9. https://doi.org/10.1046/j.13652559.1999.00650.x PMID: 10231422

2. Perera NR, Kumarasinghe MP, Sterrett GF. Hepatoid yolk sac tumour of the ovary - a case report. Ceylon J of Med Sci. 1995;38:15-7.

3. Zizi-Sermpetzoglou A, Petrakopoulou N, Nikolaidou ME, Tepelenis N, Savvaidou V, Vasilakaki T. Hepatoid carcinoma of the ovary: a case report and review of the literature. Eur J Gynaecol Oncol. 2009;30(3):341-3. PMID:19697638

4. Gopaldas R, Kunasan, R, Plymyer, Matthew R, Bloch, Robert S. Hepatoid malignancy of unknown origin--a diagnostic conundrum: review of literature and case report of collision with adenocarcinoma. Surg Oncol. 2005;14(1):1125. https://doi.org/10.1016/j.suronc.2004.09.011 PMID: 15777886

5. Prat J, Bhan AK, Dickersin GR, Robby $\mathrm{Sj}$, Scully RE. Hepatoid yolk sac tumour of the ovary: a light microscopic, ultrastructural and immunohistochemical study of seven cases. Cancer. 1982;50(11):2355-68.

6. Li XL, Wang X, Zhu FF. Hepatoid carcinoma of the ovary: A case report and review of the literature. Oncol let. 2012;4(5):947-50. https:// doi.org/10.3892/ol.2012.879 $\quad$ PMID:23162628 PMCID:PMC3499544

7. Pandey $\mathrm{M}$ and Truica C. Hepatoid carcinoma of the ovary. J Clin Oncol. 2011; 29:446 8.

8. Wang L, Zhong Y, Sun L, Zhou H, Chen W, Zhang X. Clinical and pathological features of hepatoid carcinoma of the ovary. World 
293. Hepatoid Variant of ...

J of Surg Oncol. 2013; 11:29. https://doi. org/10.1186/1477-7819-11-29 PMID:23363542 PMCID:PMC3563625

9. Pitman MB, Triratanachat S, Young RH, Oliva E. Hepatocyte paraffin 1 antibody does not distinguish primary ovarian tumors with hepatoid differentiation from metastatic hepatocellular carcinoma. Int J Gynecol Pathol. 2004;23(1):5864. PMID: 14668552

10. Hibner M, Greenspan D. Ovarian metastases from the biliary tract, pancreas and liver carci- nomas. J Gynaec Oncol. 2004;9(2):125-8.

11. Hameed $\mathrm{O}, \mathrm{Xu} \mathrm{H}$, Saddeghi S, Maluf $\mathrm{H}$. Hepatoid Carcinoma of the Pancreas. A Case Report and Literature Review of a Heterogeneous Group of Tumors. Am J Surg Pathol. 2007;31(1):146-52. https://doi.org/10.1097/01. pas.0000213370.79300.e1 PMID:17197931

12. Koswara T, Marwoto W, Siregar NC, Gumay $\mathrm{S}$, Azis H, Abdullah M, et al. Hepatoid carcinoma of the gallbladder. Acta Med Indones. 2007;39(4):179-82. PMID:18046064

\section{How to Cite This Article}

Parampalli Srinivas S, Shivamurthy A, Rao L, Gurumoorthy Bhat R. Hepatoid Variant of Yolk Sac Tumor of Both Ovaries With Widespread Intra-abdominal and Lung Metastasis: A Case Report. Iranian Journal of Pathology, 2018; 13(2): 289-293. 